\title{
Simple homotopy equivalence of nearby Lagrangians
}

\author{
by \\ Mohammed ABouzaid \\ Columbia University \\ New York, NY, U.S.A. \\ THOMAS KRAGH \\ Uppsala University \\ Uppsala, Sweden
}

\section{Introduction}

Let $N$ be a closed smooth manifold. In accordance with conjectures of $\mathrm{Arnol}^{\prime} \mathrm{d}$ and Eliashberg, the symplectic topology of the cotangent bundle $T^{*} N$ is expected to be deeply tied to the smooth topology of $N$. One way to study the symplectic topology of $T^{*} N$ is to consider its Lagrangian submanifolds; symplectic topologists have traditionally focused on those Lagrangians which are exact with respect to the canonical Liouville form on the cotangent bundle. The main result of this paper is the following.

THEOREM 1. If $L \subset T^{*} N$ is a closed exact Lagrangian, then the canonical map $L \subset T^{*} N \rightarrow N$ is a simple homotopy equivalence.

We shall presently explain our strategy for proving this result, after highlighting the following consequence: recall that a lens space is a quotient of $S^{2 n-1} \subset \mathbb{C}^{2 n}$ by a finite cyclic subgroup of $U(n)$. It was classically known, following Franz and Reidemeister, that the simple homotopy type of lens spaces determines their diffeomorphism type (a modern account is provided by Milnor in $[13, \S 12]$ ). From Theorem 1 , we therefore conclude an answer to a long-standing open question.

Corollary 1.1. A pair of lens spaces are diffeomorphic if and only if their cotangent bundles are symplectomorphic.

The proof of Theorem 1 can be broken into three different steps: first, we introduce a notion of Whitehead torsion for a Floer theoretic equivalence. Here, a Floer theoretic equivalence should be interpreted as an equivalence in the Fukaya category of a symplectic

M. A. was supported by NSF grant DMS-1308179, and by Simons Foundation grant 385571. The authors were hosted by the Mittag-Leffler Institute during its "Symplectic Geometry and Topology" program in Fall 2015. 
manifold, though our usage of this machinery is quite limited, and we give a self-contained discussion of this notion in terms of the existence of Floer cocycles whose products satisfy a certain non-degeneracy condition. At a technical level, the study of torsion invariants for equivalences is a minor generalisation of the work of Sullivan [22] and Hutching and Lee [10] who considered the torsion of acyclic Floer complexes. However, we find it convenient to provide our own account for the construction of this invariant, as the specific context we are studying allows for greatly simplified proofs, especially of the invariant of torsion under Hamiltonian isotopies. This is the content of $\S 3$.

Remark 1.2. There is a direct connection between the torsion of a Floer-theoretic equivalence and that of an acyclic Floer complex, provided by associating to an equivalence its cone, which can be considered as an immersed Lagrangian, equipped with a bounding cochain in the sense of Akaho and Joyce [3], with acyclic Floer cohomology with any other Lagrangian, so that one can apply Sullivan's techniques (see also [21]). We expect that the torsion in our sense agrees with the torsion of the Floer homology of the associated cone.

The next step is to appeal to the results of [2], [9], [11], [14], showing that every closed Lagrangian in a cotangent bundle gives rise to an object of the Fukaya category which is Floer-theoretically equivalent to the zero section. In particular, it makes sense to consider the torsion of this Floer-theoretic equivalence, and we prove that this vanishes in $\S 4$. This section is at the heart of the paper, and the key idea that it uses is a large-scale Hamiltonian deformation (see also [12]) which allows us to reduce the computation of torsion to a computation at the level of certain associated graded groups which admit a particularly simple description.

Finally, we show in $\S 5$ that the Floer-theoretic torsion which we introduce agrees, in the setting of cotangent bundles, with the classical torsion. Here, the main ingredient is the compatibility of Floer and Morse theoretic constructions in the exact setting, reminiscent of the constructions of Piunikhin-Salamon-Schwarz in [16] relating Floer and Quantum cohomology.

From the above ingredients, the proof of Theorem 1 is immediate. We make it explicit for the record.

Proof. Using [2] and [11], we see that the map $L \rightarrow N$ is a homotopy equivalence, and thus its Whitehead torsion is well defined. Proposition 5.2 shows that this Whitehead torsion agrees with the torsion of the Floer theoretic equivalence between $L$ and $N$. Proposition 4.4 implies that this torsion vanishes. 


\section{Acknowledgements.}

The first author would like to thank Shmuel Weinberger and Dennis Sullivan for explanations about the role of simple homotopy theory in the classification of manifolds. The second author would like to thank John Rognes for the same reason.

We are grateful to the referees for the extensive and helpful suggestions.

\section{Whitehead torsion}

In this section we give a crash course in Whitehead torsion and Whitehead groups. All the results are standard, and more details can be found in [24].

Let $R=\mathbb{Z}[G]$ be the group ring of a discrete group $G$, and let $C_{*}=\left(R\left[\alpha_{i}\right], d\right)$ be a finitely generated acyclic chain complex of left $R$ modules freely generated by some $\alpha_{i}$ with degree $\left|\alpha_{i}\right|$-we assume that the differential always increases the degree. The Whitehead torsion of the pair $\left(C_{*},\left\{\alpha_{i}\right\}\right)$ is defined as its equivalence class under the relations generated by

- a very simple expansion (inverse: very simple contraction) given by direct sum with the chain complex

$$
\ldots \longrightarrow 0 \longrightarrow R \stackrel{\mathrm{Id}}{\longrightarrow} R \longrightarrow 0 \longrightarrow \ldots
$$

with the obvious choice of generators;

- a handle slide given by replacing a basis element by itself plus a linear combination of the other basis elements in the same degree;

- a simple base change given by replacing any $\alpha_{i}$ with $( \pm g) \alpha_{i}$ for $g \in G$.

These equivalence classes form a group $\mathrm{Wh}(G)$ (the Whitehead group of $G$ ) under direct sum. We speak of the Whitehead torsion of a complex when the choice of basis up to these equivalences is self-evident (as it is for Floer, Morse, and simplicial complexes).

Remark 2.1. Since we are not working in characteristic 2, we point out that a choice of Morse function does not determine a basis for the Morse complex, but only a basis up to sign, because the assignment of a generator to a critical point requires a choice of orientation of the descending (or ascending, depending on conventions) manifold at the given point. The same phenomenon occurs in Floer theory, but is inconsequential for applications, because of the independence of the Whitehead class on sign changes.

Using the chosen basis (and choosing an auxiliary ordering), we can identify each differential with a matrix with coefficients in $\mathbb{Z}[G]$. Whitehead proved in $[24]$ that, using these moves, any acyclic chain complex is equivalent to one with support in any two adjacent degrees, and a single non-zero differential, which then has to be an isomorphism 
in $\mathrm{Gl}_{n}(\mathbb{Z}[G])$. The inverse in the Whitehead group can be represented by this 2 -term complex with the inverse differential (see [13] for details).

For any chain homotopy equivalence $f:\left(C, d_{C}\right) \rightarrow\left(D, d_{D}\right)$ of left $\mathbb{Z}[G]$-module chain complexes (with bases), we can define the mapping cone as

$$
(C[1] \oplus D, d)=\left(\begin{array}{cc}
d_{C}[1] & f \\
0 & d_{D}
\end{array}\right)
$$

(with the direct sum basis). Here, $C[1]$ means the chain complex shifted up by 1 in degree and changing the sign on the differential. This is an acyclic chain complex, and so defines an element $\tau(f)$ in $\mathrm{Wh}(G)$. An important property of this that we will use repeatedly is that

$$
\tau(f \circ g)=\tau(f)+\tau(g)
$$

for the composition of chain homotopy equivalences.

Now, let $X$ and $Y$ be connected finite CW complexes and $f: X \rightarrow Y$ a cellular homotopy equivalence with $G=\pi_{1}(Y) \cong \pi_{1}(X)$. By considering the universal covering spaces $\widetilde{X} \rightarrow X$ and $\tilde{Y} \rightarrow Y$ with their natural CW structure, we get (after a choice of base-points compatible with $f$ ) an induced chain homotopy equivalence

$$
\tilde{f}_{*}: C_{*}(\tilde{X}, \mathbb{Z}) \longrightarrow C_{*}(\tilde{Y}, \mathbb{Z}) .
$$

Here, our convention is that an $n$-dimensional cell contributes a generator of degree $-n$, so that the differential raises degree as with the rest of the chain complexes we shall study. Using the choice of base-points and choosing a lift of each cell in $X$ and $Y$, this lifts to a chain homotopy equivalence of $\mathbb{Z}[G]$-module complexes (complete with choices of basis as above)

$$
\tilde{f}_{*}: C_{*}^{\mathrm{CW}}(X, \mathbb{Z}[G]) \longrightarrow C_{*}^{\mathrm{CW}}(Y, \mathbb{Z}[G])
$$

We then use the mapping cone construction on this map as above. This defines the Whitehead torsion $\tau(f)=\tau\left(\tilde{f}_{*}\right) \in \mathrm{Wh}(G)$ of $f$. This is well defined, since different choices of lifts of cells correspond to simple base changes, and changing the base-point induces isomorphisms of $\mathbb{Z}[G]$ which are well defined up to conjugation with an element $\gamma$ of $\pi_{1}(X)$. However, conjugation acts trivially on Whitehead torsion, since

$$
\left(\gamma b \gamma^{-1}\right) \sim\left(\begin{array}{cc}
\gamma b \gamma^{-1} & 0 \\
0 & 1
\end{array}\right) \sim\left(\begin{array}{cc}
b \gamma^{-1} & 0 \\
0 & \gamma
\end{array}\right) \sim\left(\begin{array}{cc}
b \gamma^{-1} \gamma & 0 \\
0 & 1
\end{array}\right) \sim(b)
$$


Remark 2.2. The main reason to work with homology is that the standard cellular cochain complex of $\tilde{X}$ is not free as a $\mathbb{Z}$-module. It is of course possible to work cohomologically starting with the linear dual of equation (2.2), but it is sometimes psychologically comforting to have direct access to the geometry of the universal cover, in contrast to abstract twisted coefficients.

The Whitehead torsion of $f$ is preserved under the notion of simple expansions and simple contractions of $X$ (or $Y$ ). To describe these let $D_{+}^{n+1}=D^{n+1} \cap\left\{x_{n+1} \geqslant 0\right\}$ be the closure of the upper half of $D^{n+1}$. This contains $D^{n}$, and we give $D^{n}$ the standard cell structure (having three cells). We extend this to a cell structure on all of $D_{+}^{n+1}$ by attaching two more cells in the obvious way.

- A simple expansion is given by attaching $D_{+}^{n+1}$ to $X$ along a cellular map $D^{n} \rightarrow X$. Effectively adding the two cells in $D_{+}^{n+1}-D^{n}$.

The inverse of this is called a simple contraction. Any map homotopic to a composition of such moves is called a simple homotopy equivalence. Whitehead proved that a map $f$ as above is a simple homotopy equivalence if and only if its Whitehead torsion is trivial.

Whitehead torsion does not generally behave well when it comes to filtrations and spectral sequences, but we will need a very special case of using a spectral sequence to compute Whitehead torsion: the context is a complex $C$ equipped with a basis $B$, with differential of the form

$$
d=\left(\begin{array}{cc}
d_{0} & d_{01} \\
0 & d_{1}
\end{array}\right),
$$

with respect to a decomposition $B=B_{0} \cup B_{1}$ of this basis.

Lemma 2.3. (Cf. [13, Theorem 5.2]) If $C_{0}$ and $C_{1}$ are both acyclic with trivial Whitehead torsion with respect to $B_{0}$ and $B_{1}$, then $C$ is acyclic with trivial Whitehead torsion with respect to $B$.

Proof. The Whitehead moves corresponding to a very simple expansion, handle slide, or simple base change for the basis of $C_{0}$ give rise to the corresponding moves for the basis of $C$. In contrast, note that a very simple contraction of $C_{0}$, which corresponds to eliminating a summand with differential

$$
\left(\begin{array}{ll}
0 & 1 \\
0 & 0
\end{array}\right)
$$

does not directly correspond to a contraction on $C$, because the entries of the differential matrix to the right of this $2 \times 2$ block may be non-empty. However, assuming that the 
differential on $C_{0}$ contains such a block, we see that

$$
d(x)=y+\sum_{i=1}^{k} c_{k} b_{k}, \quad c_{k} \in R,
$$

where $x$ and $y$ are the basis elements of $C_{0}$ in question and the $b_{k}$ are the basis elements of $C_{1}$. This means that we may perform a handle slide and change $y$ into $y^{\prime}=d(x)$, which does not change the upper triangular block structure (nor does it change the two diagonal blocks), but it does change the row associated with $x$ into a row with only 0 's and a single 1 . This $y^{\prime}$ has $d\left(y^{\prime}\right)=0$, since the complex is acyclic. We may now perform the contraction on $C$. So, inserting this extra move in front of all very simple contractions provides a reduction of all of $C$ to $C_{1}$.

Applying the above argument inductively, we conclude the following.

Corollary 2.4. Let $0=F^{0} C \subset \ldots \subset F^{k+1} C=C$ be a filtered complex of free left $\mathbb{Z}[G]$ modules, equipped with a basis that is compatible with the filtration (i.e. that descends to a basis of the associated graded complexes).

If each filtered quotient $F^{p} C / F^{p-1} C$ is acyclic, and has trivial Whitehead torsion with respect to the induced basis, then the based complex $C$ has trivial Whitehead torsion.

The following lemma is not immediately clear from the definition, but will be needed to prove perturbation invariance in Floer and Morse theoretic contexts. For a proof, see [13, Lemma 7.7]

Lemma 2.5. If $f, g: C_{*} \rightarrow D_{*}$ are chain homotopic maps (as left $\mathbb{Z}[G]$-modules), then their Whitehead torsions agree.

\section{Whitehead torsion and the Fukaya category}

Whitehead groups have been defined and used before in the context of intersection Floer homology; the work by M. Sullivan in [22] is the most relevant for this paper (see also [10] which studies a much harder problem). Our treatment will however be essentially self-contained, in part because we will discuss torsion for Floer theoretic equivalences, but also because we will only consider such groups in the exact setting. This means that the action filtration will allow us to use Corollary 2.4 to give a much shorter proof of the invariance of Whitehead torsion. We shall use Seidel's book [17] as reference for various points in Lagrangian Floer theory. 


\subsection{Floer cohomology and the action filtration}

We begin by considering the following context: let $(M, \lambda)$ be a Liouville domain with symplectic form $\omega=d \lambda$, equipped with a grading $\widetilde{G r}_{\Lambda}(M)$ and a background class $b \in H^{2}\left(M, \mathbb{Z}_{2}\right)$. Here, the grading is a choice of cover of the Grassmannian bundle of Lagrangian subspaces in $T M$, which restricts over each point in $M$ to a universal cover for the Grassmannian of linear Lagrangians (see $[17, \S 11 \mathrm{~h}]$ ). To simplify the exposition, we shall assume throughout that $b$ is the second Stiefel-Whitney class of an orientable vector bundle $V$ on $M$.

Remark 3.1. If $N$ is a smooth manifold, and $M$ its cotangent bundle with projection map $\pi: T^{*} N \rightarrow N$, there is a preferred grading characterized by the property that the tangent directions to the cotangent fibres are a section of the corresponding family of universal covers of the Grassmannian bundle.

We shall consider the pullback of $w_{2}(N)$ as background class on $T^{*} N$. This is the second Stiefel-Whitney class of the orientable bundle $T N \oplus(\operatorname{det}(T N))^{\oplus 3}$, as can be seen by computing the total Stiefel-Whitney class

$$
\begin{aligned}
(1+ & \left.w_{1}(N)+w_{2}(N)+\ldots\right)\left(1+w_{1}(N)\right)^{3} \\
& =\left(1+w_{1}(N)+w_{2}(N)+\ldots\right)\left(1+w_{1}(N)+w_{1}^{2}(N)+\ldots\right) \\
& =\left(1+w_{2}(N)+\ldots\right) .
\end{aligned}
$$

Consider an exact Lagrangian $L$ which is a brane relative to the background class $b$ : this means that we fixed a function $f: L \rightarrow \mathbb{R}$ such that $d f=\left.\lambda\right|_{L}$, a lift of the Gauss map of $L$ to $\widetilde{\operatorname{Gr}}_{\Lambda}(M)$, as well as a relative Spin structure, i.e. a Spin structure on the direct sum of the tangent space of $L$ with the restriction of the vector bundle $V$. The first structure implies that the Lagrangian is exact, the second that it has vanishing Maslov class, and the last that the second Stiefel-Whitney class agrees with the restriction of $b$.

The ambiguity in the choice of primitive is $H^{0}(L, \mathbb{R})$, corresponding to adding a locally constant function, the ambiguity in the choice of grading is $H^{0}(L, \mathbb{Z})$, corresponding to the fact that the fundamental group of the Grassmannian of linear Lagrangians is $\mathbb{Z}$, and the ambiguity in the choice of relative Spin structure is $H^{1}\left(L, \mathbb{Z}_{2}\right)$.

Remark 3.2. By definition, the Liouville form vanishes on the zero section of $T^{*} N$. Since the tangent space of the zero section is transverse to the cotangent fibres, it admits a lift to the preferred covering of the Grassmannian bundle. Moreover, the background class on $T^{*} N$ was chosen so that the zero section is relatively Spin with a preferred relative Spin structure. Indeed, the computation of equation (3.1) is universal, so that we can fix a Spin structure on the direct sum of two copies of the universal bundle over 
BO with three copies of its orientation line, and pull this Spin structure back to $N$ to define a relative spin structure of $T N$ relative to $T N \oplus \operatorname{det}(T N)^{3}$.

If $L \subset T^{*} N$ is an exact Lagrangian, then a primitive $f$ of the restriction $\left.\lambda\right|_{L}$ exists by definition. As a consequence of [11], every such Lagrangian has vanishing Maslov class, and thus admits a lift to the fibrewise universal cover of the Grassmannian bundle. Moreover, as a consequence of [2], the map $L \rightarrow N$ is a homotopy equivalence. This implies that the Stiefel-Whitney classes of $L$ agree with the pullback of the StiefelWhitney classes of $N$ under this map, and hence $L$ admits a relative Spin structure (while this goes back to Thom [23], we would like to point out Nash's proof [15], which is much more elementary than the standard proof due to Atiyah [4]).

If $\left(L_{0}, f_{0}\right)$ and $\left(L_{1}, f_{1}\right)$ are transverse branes, we may associate with each intersection point $x \in L_{0} \cap L_{1}$ a Maslov index $\operatorname{deg}(x) \in \mathbb{Z}$ and a graded 1-dimensional real vector space, which we denote by $o_{x}$ as in $[17, \S 12 \mathrm{f}]$ (see also [1, Appendix A]), so that, whenever $J$ is a generic $\omega$-tame almost complex structure on $M$, the following holds: for each pair $(x, y)$ of intersection points such that $\operatorname{deg}(y)+1=\operatorname{deg}(x)$, every element $u$ of the moduli space $\mathcal{M}(x, y)$ of $J$-holomorphic strips induces a canonical isomorphism

$$
\varkappa_{u}: \mathrm{O}_{y} \longrightarrow \mathrm{o}_{x}
$$

More precisely, we define $\mathcal{M}(x, y)$ to be the quotient by $\mathbb{R}$-translation of the space of all the holomorphic maps $u: \mathbb{R} \times[0,1] \rightarrow M$ with boundary conditions $u(\mathbb{R} \times\{0\}) \subset L_{0}$ and $u(\mathbb{R} \times\{1\}) \subset L_{1}$, and with the asymptotic conditions that $u \rightarrow x$ as $s \rightarrow-\infty$ and $u \rightarrow y$ as $s \rightarrow \infty$. The Lagrangian Floer cochains are then defined to be the direct sum

$$
C F^{*}\left(L_{0}, L_{1} ; \mathbb{Z}\right)=\bigoplus_{x \in L_{0} \cap L_{1}}\left|\mathrm{o}_{x}\right|,
$$

where $\left|o_{x}\right|$ is the orientation line of $o_{x}$ (i.e. the free Abelian group generated by the two orientations of this vector space, with the relation that their sum vanishes). The differential is the sum, over all $u \in \mathcal{M}(x, y)$, of the maps on orientation lines induced by equation (3.2).

By Stokes's theorem, the symplectic area $\int u^{*} \omega$ of $u \in \mathcal{M}(x, y)$ (which is positive, since $u$ is holomorphic) is

$$
f_{1}(x)-f_{1}(y)+f_{0}(y)-f_{0}(x) \geqslant 0 \quad \Longrightarrow \quad f_{1}(x)-f_{0}(x) \geqslant f_{1}(y)-f_{0}(y) .
$$

We may therefore associate with each intersection point its action

$$
\mathcal{A}(x)=f_{1}(x)-f_{0}(x),
$$


and conclude that the moduli space $\mathcal{M}(x, y)$ is empty whenever $\mathcal{A}(x) \leqslant \mathcal{A}(y)$ and $x \neq y$. At the level of Floer complexes, we obtain a filtration by subcomplexes

$$
F^{a} C F^{*}\left(L_{0}, L_{1} ; \mathbb{Z}\right)=\bigoplus_{\mathcal{A}(x) \geqslant a}\left|\mathrm{o}_{x}\right| .
$$

The reader should keep in mind that, with the conventions we have adopted, the differential raises action.

Whenever $L=L_{0}=L_{1}$, we follow Biran-Cornea [6], Seidel [18] and Sheridan [19], and pick a Riemannian metric together with a Morse-Smale function $h: L \rightarrow \mathbb{R}$ with a unique minimum, and define

$$
C F^{*}(L, L ; \mathbb{Z})=C M^{*}(L, h ; \mathbb{Z})
$$

where the right-hand side are Morse cochains with differential given by counting negative gradient flow lines. The minimum of $h$ defines a cocycle $e \in C M^{0}(L, h ; \mathbb{Z})$ which, at the level of cohomology, corresponds to the unit in $[e] \in H^{0}(L, \mathbb{Z})$ under the isomorphism of Morse cohomology with ordinary cohomology. We set the action filtration on $C F^{*}(L, L ; \mathbb{Z})$, so that all generators have zero action.

\subsection{The product on Floer cohomology}

Given a triple of Lagrangian branes $\left(L_{0}, L_{1}, L_{2}\right)$, which are in transverse position, we have a cochain map

$$
C F^{*}\left(L_{1}, L_{2} ; \mathbb{Z}\right) \otimes C F^{*}\left(L_{0}, L_{1} ; \mathbb{Z}\right) \stackrel{\cdot}{\longrightarrow} C F^{*}\left(L_{0}, L_{2} ; \mathbb{Z}\right)
$$

given by a count of rigid triangles. More precisely, say that $\left(J_{01}, J_{12}, J_{02}\right)$ are a triple of almost complex structures used to define the above Floer complexes. Choosing a (generic) family $J$ of almost complex structures smoothly parameterized by the disc, which agrees at three distinct marked points on the boundary (ordered clockwise) with the above triple, we obtain a moduli space $\mathcal{M}\left(x_{0}, x_{1}, x_{2}\right)$ associated with every triple of intersection points $x_{0} \in L_{0} \cap L_{2}, x_{1} \in L_{0} \cap L_{1}$ and $x_{2} \in L_{1} \cap L_{2}$ as follows: elements of $\mathcal{M}\left(x_{0}, x_{1}, x_{2}\right)$ are finite-energy maps from the disc to $M$, which are holomorphic in the interior, mapping the three marked points to $x_{0}, x_{1}$ and $x_{2}$, with boundary conditions along the boundary given by the triple of Lagrangians $L_{0}, L_{1}$ and $L_{2}$ (see Figure 1 ). Stokes's theorem shows that the symplectic area $\int u^{*} \omega$ of an element $u \in \mathcal{M}\left(x_{0}, x_{1}, x_{2}\right)$ disc is given by

$$
f_{0}\left(x_{1}\right)-f_{0}\left(x_{0}\right)+f_{1}\left(x_{2}\right)-f_{1}\left(x_{1}\right)+f_{2}\left(x_{0}\right)-f_{2}\left(x_{2}\right)=\mathcal{A}\left(x_{0}\right)-\mathcal{A}\left(x_{1}\right)-\mathcal{A}\left(x_{2}\right) .
$$




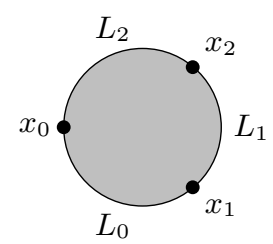

Moduli space defining the product

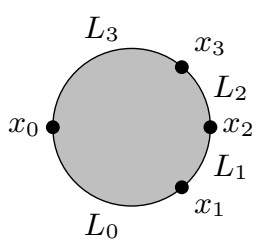

Moduli space defining the homotopy

Figure 1. Discs with marked points and boundary conditions
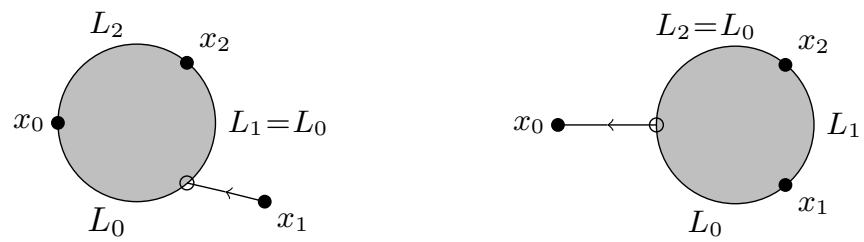

Figure 2. Discs with gradient trajectory attached

Using coordinates $(s, t)$ on the disc, the symplectic area is given by

$$
\int \omega\left(\partial_{s} u, \partial_{t} u\right) d s \wedge d t=\int \omega\left(\partial_{s} u, J_{s, t} \partial_{s} u\right) d s \wedge d t
$$

where $J_{s, t}$ is the restriction of $J$ to the point with coordinates $(s, t)$. The right-hand side is positive, because $J_{s, t}$ is tame, so we conclude that the product map is filtered when the filtration on the tensor product is given by defining the action of a generator $\alpha \otimes \beta$ to be the sum $\mathcal{A}(\alpha)+\mathcal{A}(\beta)$.

Whenever two of these Lagrangians agree, we instead count configurations consisting of holomorphic discs and perturbed gradient flow lines for some generic Morse-Smale function with a unique minimum. Explicitly, the flow lines are maps from $(-\infty, 0]$ or $[0, \infty)$ to the given Lagrangian, which satisfy the gradient flow equation outside a compact set, and a perturbation of this equation (by a generically chosen vector field) near the endpoint zero. The direction of the gradient flow line depends on what side of the equation the Floer group is on-see Figure 2, where the arrow indicates positive gradient flow. This product is also filtered with our conventions.

Remark 3.3. Choosing a perturbation on the gradient flow lines allows us to ensure that the moduli spaces described in Figure 2 are regular. If we desire to work only with unperturbed flow lines, we need to make sure that the critical points are distinct from the intersection points of Lagrangians.

Applying this to the special case when $L_{1}=L_{2}$, and two choices of almost complex 
structures $J_{01}$ and $J_{01}^{\prime}$, we obtain a map

$$
C M^{*}\left(L_{1}, L_{1} ; \mathbb{Z}\right) \otimes C F^{*}\left(L_{0}, L_{1}, J_{01} ; \mathbb{Z}\right) \longrightarrow C F^{*}\left(L_{0}, L_{1}, J_{01}^{\prime} ; \mathbb{Z}\right),
$$

where we temporarily introduce notation which incorporates the choice of almost complex structure. If we input the class of the minimum in $C M^{*}\left(L_{1}, L_{1} ; \mathbb{Z}\right)$, we obtain a map

$$
C F^{*}\left(L_{0}, L_{1}, J_{01} ; \mathbb{Z}\right) \longrightarrow C F^{*}\left(L_{0}, L_{1}, J_{01}^{\prime} ; \mathbb{Z}\right)
$$

which is a continuation map for almost complex structures.

LEMMA 3.4. The continuation map for almost complex structures is a chain isomorphism.

Proof. The computation of equation (3.7) applies verbatim to show that the energy of continuation maps is positive, and hence, at the chain level, the map is upper triangular with respect to the action filtration. It therefore suffices to show that the entries along the diagonal are \pm 1 . These entries count constant holomorphic discs at the intersection points of $L_{0}$ with $L_{1}$ (together with a negative gradient flow line to the unique minimum, which can be ignored in the generic case where the intersection points all flow to this minimum).

More generally, one can show that the unit $e \in H F^{0}(L, L ; \mathbb{Z})$ acts as a 2-sided identity for the product.

Up to a sign change, the product is part of the $A_{\infty}$ structure on the Fukaya category, as discussed in $[17, \S 12]$. We shall only need the fact that it is homotopy associative. Concretely, the count of holomorphic discs with boundary mapping to a quadruple of Lagrangian branes $\left(L_{0}, L_{1}, L_{2}, L_{3}\right)$ as in Figure 1 defines a homotopy for the diagram

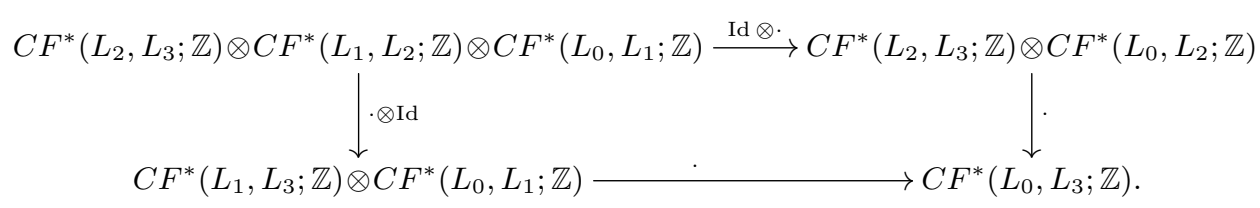

This homotopy again preserves the action filtration, since Stokes's theorem shows that $\mathcal{A}\left(x_{1}\right)+\mathcal{A}\left(x_{2}\right)+\mathcal{A}\left(x_{3}\right)-\mathcal{A}\left(x_{0}\right)$ is negative.

Definition 3.5. A pair of Lagrangians $L_{0}$ and $L_{1}$ are Floer theoretically equivalent if there exist cocycles $\alpha \in C F^{i}\left(L_{0}, L_{1} ; \mathbb{Z}\right)$ and $\beta \in C F^{-i}\left(L_{1}, L_{0} ; \mathbb{Z}\right)$ such that

$$
\begin{aligned}
& {[\beta] \cdot[\alpha]=e_{0} \in H F^{0}\left(L_{0}, L_{0} ; \mathbb{Z}\right),} \\
& {[\alpha] \cdot[\beta]=e_{1} \in H F^{0}\left(L_{1}, L_{1} ; \mathbb{Z}\right),}
\end{aligned}
$$

where $e_{i}$ denotes the unit. 

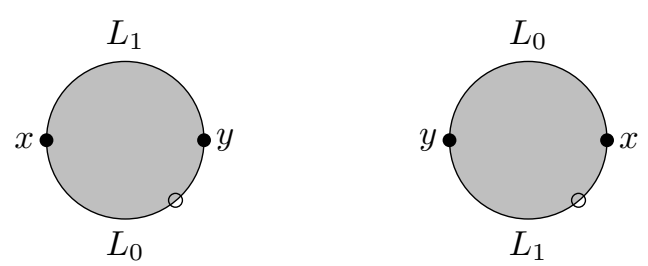

Figure 3. The circled point at the boundary goes through a fixed point on the Lagrangian. Fixing where this point is on the boundary component corresponds to gauging the action of $\mathbb{R}$.

The cocycles $\alpha$ and $\beta$ are called (Floer theoretic) equivalences.

Remark 3.6. Whenever $L_{0}$ and $L_{1}$ are connected, the possible cohomology classes of all Floer theoretic equivalences are uniquely determined up to sign, because they induce isomorphisms

$$
H F^{*+i}\left(L_{0}, L_{1} ; \mathbb{Z}\right) \cong H F^{*}\left(L_{0}, L_{0} ; \mathbb{Z}\right) \cong H F^{*-i}\left(L_{1}, L_{0} ; \mathbb{Z}\right),
$$

and the group $H F^{*}\left(L_{0}, L_{0} ; \mathbb{Z}\right)$ is isomorphic to $H^{*}(L ; \mathbb{Z})$, which is of rank 1 in degree 0 .

Remark 3.7. In the cotangent bundle case we know that any brane supported on a closed Lagrangian $L \subset T^{*} N \rightarrow N$ is Floer theoretically equivalent to a rank-1 local system on the zero section, by the results of [2]. As we are working over $\mathbb{Z}$, and rank-1 local systems are in bijective correspondence with relative Spin structures whenever the latter exist, this implies that there is a relative Spin structure on $L$ so that the corresponding object is Floer theoretically equivalent to the zero section equipped with the relative Spin structure fixed in Remark 3.2.

When dealing with such a Lagrangian in later sections we will always fix the brane structure in this way.

Assuming for simplicity that $\alpha$ and $\beta$ are represented by unique intersection points $x$ and $y$, the condition that $L_{0}$ and $L_{1}$ are Floer-theoretically equivalent can be restated as the fact that the signed count of elements of $\mathcal{M}(x, y)$ whose boundary passes through any fixed point of $L_{0}$ is 1 , as is the count of such elements passing through a fixed point of $L_{1}$ (see Figure 3 ). In the general case, one requires instead that a linear combination of such counts equal 1 .

It follows immediately from the associativity of multiplication at the cohomological level that Floer theoretic equivalence is transitive. We record a precise formulation for future use.

LemMA 3.8. If $\left(\alpha_{1}, \beta_{1}\right)$ and $\left(\alpha_{2}, \beta_{2}\right)$ are Floer theoretic equivalences for pairs $\left(L_{0}, L_{1}\right)$ and $\left(L_{1}, L_{2}\right)$, then $\left(\alpha_{2} \cdot \alpha_{1}, \beta_{1} \cdot \beta_{2}\right)$ are Floer theoretic equivalences for the pair $\left(L_{0}, L_{2}\right)$ assuming they are transverse or equal. 
To see that Hamiltonian isotopies induce Floer theoretic equivalences, we start by considering the following special case.

Lemma 3.9. Assume that $K_{1} \subset M$ and $K_{0} \subset M$ are exact Lagrangians and that $W=D_{\varepsilon} T^{*} K_{0}$ is a Weinstein neighbourhood of $K_{0}$ containing $K_{1}$ as the graph of the differential of a Morse function $g: K_{0} \rightarrow \mathbb{R}$. Then, $K_{1}$ and $K_{0}$ are Floer theoretically equivalent.

Proof. It suffices to prove that the graph of an exact 1-form is Floer theoretically equivalent to the zero section. This goes back to Floer: in [8], he shows that, by picking $J_{01}$ and $J_{10}$ carefully, we get isomorphisms of complexes

$$
C F^{*}\left(K_{0}, K_{1} ; \mathbb{Z}\right) \cong C M^{*}(g ; \mathbb{Z}) \text { and } C M^{*}(-g ; \mathbb{Z}) \cong C F^{*}\left(K_{1}, K_{0} ; \mathbb{Z}\right)
$$

The Floer theoretic equivalence $\alpha \in C F^{0}\left(K_{0}, K_{1} ; \mathbb{Z}\right)$ is then the sum of the intersections corresponding to the local minima of $g$, and $\beta \in C F^{0}\left(K_{1}, K_{0} ; \mathbb{Z}\right)$ corresponds to the sum of all the local maxima of $g$. Indeed, the product $\alpha \cdot \beta$ is a count of holomorphic strips (corresponding to gradient trajectories of $g$ from a minimum to a maximum), which pass through the unique minimum of some other generic Morse function on $f: K_{0} \rightarrow \mathbb{R}$, and this count is exactly 1. Using continuation maps as in Lemma 3.4, we conclude the desired result for any generic choice of almost complex structure.

Corollary 3.10. Assume that $K_{0}$ and $K_{1}$ are Hamiltonian isotopic and transverse or equal. Then, $K_{0}$ and $K_{1}$ are Floer theoretically equivalent.

Proof. Any Hamiltonian isotopy can be perturbed relative its endpoints, so that it can be expressed as a concatenation of the special case in Lemma 3.9. Furthermore, this can be done such that all the intermediate Lagrangians are pairwise transverse and transverse to $K_{0}$ and $K_{1}$. This means that these equivalences can be composed as in Lemma 3.8 to get the desired equivalence.

\subsection{Floer chains with coefficients in the group ring of $M$}

In this section we consider local coefficients on Floer homology, which was first done in [5], and later in [7] and [1].

We now fix a base-point $\star \in M$ and consider the local coefficient system $R$ on $M$ whose value at a point $x$ is the free Abelian group $R_{x}$ on the components of the space of paths from $\star$ to $x$. The fibre at the base point is the group ring $R_{\star}=\mathbb{Z}\left[\pi_{1}(M, \star)\right]$, which acts on all other fibres by concatenation. We shall define a Floer homology group with values in the coefficient system $R$. 
Given transverse Lagrangian branes $L_{0}$ and $L_{1}$ as before, the underlying graded $R_{\star}$ module for this vector space is

$$
C F_{-i}\left(L_{0}, L_{1} ; R\right)=\bigoplus_{\substack{x \in K \cap L \\ \operatorname{deg}(x)=i}}\left|\mathrm{o}_{x}\right|^{\vee} \otimes R_{x},
$$

where $\left|\mathrm{o}_{x}\right|^{\vee}$ is the linear dual of the graded line $\left|\mathrm{o}_{x}\right|$ appearing in equation (3.3). The differential is defined as before by counting elements of moduli spaces of rigid strips $\mathcal{M}(x, y)$, noting that a map $u$ representing such an element induces, by concatenation with the path $u(\mathbb{R} \times\{t\})$, an $R_{\star}$-linear map

$$
R_{x} \longrightarrow R_{y}
$$

Taking the tensor product with the map induced by equation (3.2) on dual orientation lines, we obtain

$$
\partial_{u}:\left|o_{x}\right|^{\vee} \otimes R_{x} \longrightarrow\left|o_{y}\right|^{\vee} \otimes R_{y} .
$$

By construction, Floer chains are equipped with a filtration by subcomplexes

$$
F^{a} C F_{*}\left(L_{0}, L_{1} ; R\right)=\bigoplus_{\substack{x \in L_{0} \cap L_{1} \\ \mathcal{A}(x) \leqslant-a}}\left|\mathrm{o}_{x}\right|^{\vee} \otimes R_{x} .
$$

We extend this definition as before to the case $L_{0}=L=L_{1}$ by picking a Morse function $h$ on $L$, and defining

$$
C F_{*}(L, L ; R)=C M_{-*}(h ; R) .
$$

In other words, a generator of this complex corresponds to a choice of a homotopy class of paths from a critical point of $h$ to the base point in $M$. The filtration on the self-Floer group is trivial, i.e. supported at the zero level.

The construction of operations on the Fukaya category naturally extends to give rise to an $A_{\infty}$ bimodule structure on the Floer chains $C F_{*}\left(L_{0}, L_{1} ; R\right)$ over the $A_{\infty}$ algebras $C F^{*}\left(L_{i}, L_{i}, \mathbb{Z}\right)$. We will only need a small part of this structure, which we now explicitly describe. Of key importance are the first two structure maps $(\varrho$ and $\lambda$ stand for right and left multiplication)

$$
\begin{aligned}
& C F^{*}\left(L_{0}, L_{1} ; \mathbb{Z}\right) \otimes C F_{*}\left(L_{0}, L_{2} ; R\right) \stackrel{\lambda}{\longrightarrow} C F_{*}\left(L_{1}, L_{2} ; R\right) \\
& C F_{*}\left(L_{2}, L_{0} ; R\right) \otimes C F^{*}\left(L_{1}, L_{0} ; \mathbb{Z}\right) \stackrel{\varrho}{\longrightarrow} C F_{*}\left(L_{2}, L_{1} ; R\right) .
\end{aligned}
$$

These maps are induced by the moduli spaces of holomorphic triangles as follows: given intersection points $x_{0} \in L_{0} \cap L_{2}, x_{1} \in L_{0} \cap L_{1}$ and $x_{2} \in L_{1} \cap L_{2}$, any element of the moduli 


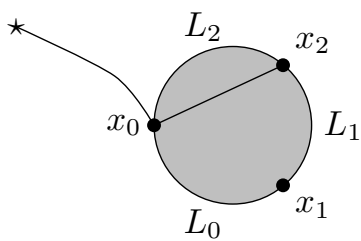

Figure 4. Contribution to local coefficients from disc. The homotopy class of a path from $\star$ to $x_{0}$ defines a generator in $R_{x_{0}}$ and composing with the homotopy class of the path from $x_{0}$ to $x_{2}$ in the image of the disc defines a map to $R_{x_{2}}$. The arc in the disc is meant to help the reader keep track of the intersection points of Lagrangians which are equipped with twisted coefficients.

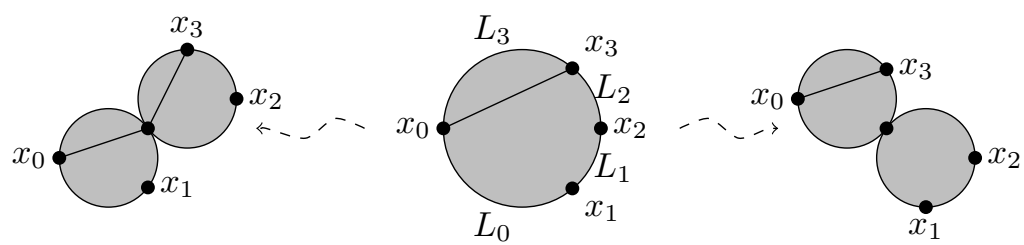

Figure 5. Breaking of discs corresponding to the two ways around the diagram in equation (3.18).

space $\mathcal{M}\left(x_{0}, x_{1}, x_{2}\right)$ determines a canonical homotopy class of paths from $x_{0}$ to $x_{2}$. Left and right multiplication are then defined by taking the tensor product of the induced map $R_{x_{0}} \rightarrow R_{x_{2}}$ with the map induced on orientation lines. By construction and Stokes's theorem, these are maps of filtered complexes.

The right action is compatible with the product in the sense that, given a quadruple $\left(L_{0}, L_{1}, L_{2}, L_{3}\right)$, the count of holomorphic discs with four marked points defines a filtered homotopy in the following diagram:

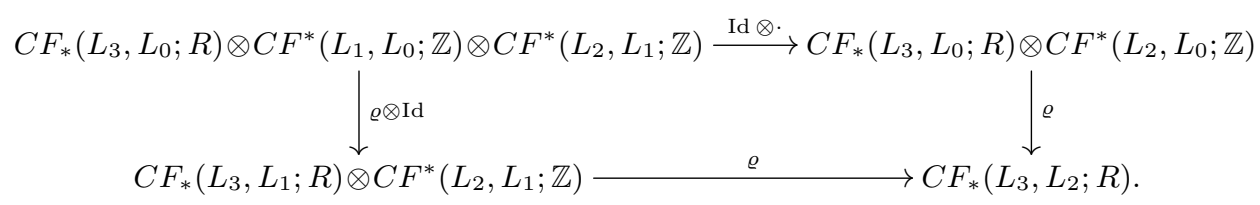

The proof is the same as the proof of homotopy associativity of the product; the only additional ingredient is the fact that the breaking of holomorphic discs is compatible with concatenation of paths as shown in Figure 5.

The left action is similarly compatible via a diagram which we omit. Finally, we shall use the fact that the left and right actions are homotopically compatible with each other, i.e. we obtain a bimodule by passing to cohomology. Concretely, this is given by 


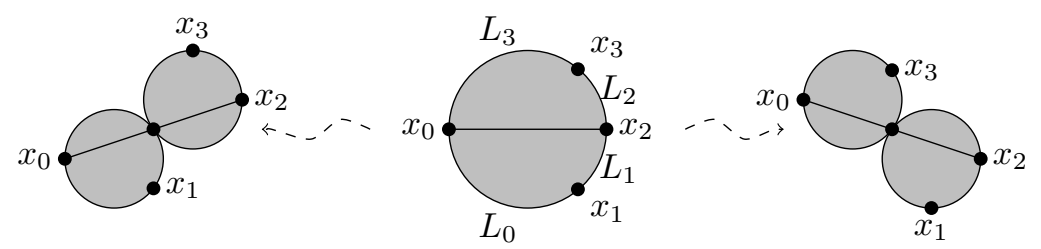

Figure 6. Breaking of discs corresponding to the two ways around the diagram in equation (3.19).

the homotopy in the diagram

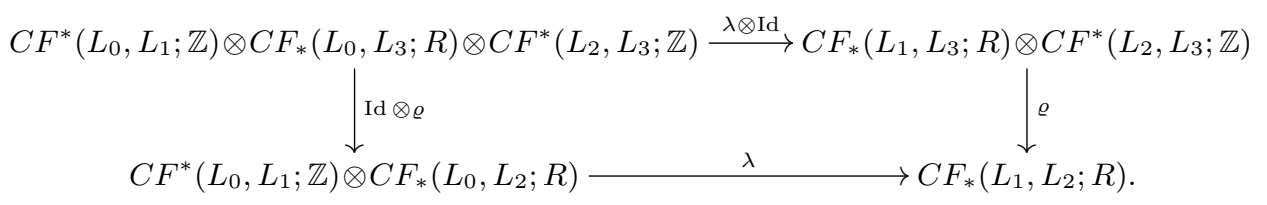

The breaking of discs which shows that this is defined with local coefficients is illustrated in Figure 6.

An easy application of Stokes's theorem shows that

$$
\mathcal{A}(\varrho(m \otimes x)) \leqslant \mathcal{A}(m)-\mathcal{A}(x) \quad \text { and } \quad \mathcal{A}(\lambda(x \otimes m)) \leqslant \mathcal{A}(m)-\mathcal{A}(x) .
$$

So, to make sense of these as filtered maps, we define action on generators of the source of $\varrho$ and $\lambda$ by $\mathcal{A}(m \otimes x)=\mathcal{A}(x \otimes m)=\mathcal{A}(m)-\mathcal{A}(x)$. Defining the action of generators of tensor products of Floer complexes to be the difference between the action of the homological element and the actions of the cohomological elements, we see that the homotopies in the above diagrams also preserve the filtrations.

\subsection{The torsion of a Floer theoretic equivalence}

Consider a pair of connected Lagrangians $K$ and $L$, and cocycles $\alpha \in C F^{*}(K, L ; \mathbb{Z})$ and $\beta \in C F^{*}(L, K ; \mathbb{Z})$ which give rise to a Floer theoretic equivalence.

Using the bimodule structure, we obtain chain homotopy equivalences

$$
\begin{gathered}
\varrho(-\otimes \alpha): C F_{*}(Q, K ; R) \longrightarrow C F_{*}(Q, L ; R), \\
\varrho(-\otimes \beta): C F_{*}(Q, L ; R) \longrightarrow C F_{*}(Q, K ; R),
\end{gathered}
$$

which are quasi-inverses, since the homotopy commutativity of the diagram in equation (3.18) shows that their compositions are homotopic to multiplication with the unit element. Multiplication with the unit is the identity, since the rigid discs that one counts (as the first in Figure 2) are constant on the disc part. Similarly, for the maps induced by $\lambda(\alpha \otimes-)$ and $\varrho(\beta \otimes-)$. 
Definition 3.11. The Floer-theoretic torsion of a pair $(K, L)$ of Floer-theoretically equivalent Lagrangians is the Whitehead torsion element in $\operatorname{Wh}\left(\pi_{1}(M, \star)\right)$ of the map

$$
C F_{*}(L, L ; R) \longrightarrow C F_{*}(K, K ; R)
$$

induced by left and right multiplication by equivalences.

Explicitly, if $\alpha \in C F^{*}(L, K ; \mathbb{Z})$ and $\beta \in C F^{*}(K, L ; \mathbb{Z})$ are equivalences, then we consider the composition

$$
C F_{*}(L, L ; R) \stackrel{\lambda(\alpha \otimes-)}{\longrightarrow} C F_{*}(K, L ; R) \stackrel{\varrho(-\otimes \beta)}{\longrightarrow} C F_{*}(K, K ; R) .
$$

LEMma 3.12. The Whitehead torsion of $\lambda(\alpha \otimes-)$ is independent of the choice of Floer theoretic equivalence and almost complex structure. Similarly for the map $\varrho(-\otimes \alpha)$.

Proof. Changing $\alpha$ to $\alpha+d \alpha^{\prime}$ : the map $\lambda\left(\alpha^{\prime} \otimes-\right)$ provides a chain homotopy between $\lambda(\alpha \otimes-)$ and $\lambda\left(\left(\alpha+d \alpha^{\prime}\right) \otimes-\right)$, which by Lemma 2.5 does not change the Whitehead torsion. By Remark 3.6, the cohomology class a Floer theoretic equivalence is uniquely determined up to sign, which does not affect the torsion.

The continuation map for complex structures is defined using the first picture in Figure 2. The resulting matrix, with respect to the action filtration, is upper triangular with entries 1 along the diagonal as in the proof of Lemma 3.4. Combined with Corollary 2.4, we see that the Whitehead torsion of the continuation map is trivial. Since composing $\lambda(\alpha \otimes-)$ with a continuation map gives a chain homotopic map, Lemma 2.5 and equation (2.1) prove that the torsion is independent of the choice of almost complex structure.

Corollary 3.13. The Floer-theoretic torsion is independent of the choice of equivalences and almost complex structures.

Remark 3.14. Traditional proofs of invariance of Whitehead torsion often rely at some point on checking invariance under handle slides, which correspond to the appearance of a flow line between critical points of equal index, which is generic in a 1-parametric family. The above result covers the analogue in Floer theory.

\subsection{Torsion is Hamiltonian invariant}

We shall presently strengthen Lemma 3.12 by showing that torsion is in fact invariant under Hamiltonian isotopies. To this end, we consider Hamiltonian isotopic Lagrangians $K_{0}$ and $K_{1}$, and a fixed Lagrangian $L$. 
We start by considering the special case of Lemma 3.9, where we additionally assume that both Lagrangians $K_{0}$ and $K_{1}$ are transverse to $L$. Let $g: K_{0} \rightarrow \mathbb{R}$ be the Morse function defining $K_{1}$, as in Lemma 3.9. In the Weinstein neighourhood $D_{\varepsilon} T^{*} K_{0}$, we have a standard isotopy given by the graph of $t d g$ for $t \in[0,1]$.

LEMma 3.15. The following properties hold for a generic $C^{1}$-small perturbation of the isotopy between $K_{0}$ and $K_{1}$ :

(1) there are finitely many points $t \in I$ such that $K_{t} \cap L$ is non-transverse;

(2) for each $t \in I$ there are finitely many points in $K_{t} \cap L$;

(3) $K_{t}$ and $K_{s}$ are transverse for $t \neq s$.

Proof. The desired properties hold for $t$ and $s$ close to 0 and 1, by the transversality assumption. Any $C^{1}$ small perturbation of the isotopy (on a compact set in the interior of $\left.K_{0} \times I\right)$ can be realized as the differentials of $h_{t}(x) g(x), t \in I$, where $h_{t}(x)=t$ when $t$ is close to 0 and 1 . This satisfies (3) as long as $h_{t}(x)$ has positive derivative with respect to $t$. Standard transversality arguments thus show that we can pick $h_{t}$ with such positive derivative and such that the isotopy and $L$ are transverse. This implies that the locus of intersection, say $S \subset K_{0} \times I$, is a 1-dimensional manifold. Now, (1) and (2) will be satisfied if the restriction of the projection to $I$, say $p: S \rightarrow I$, is a Morse function. To see that we may perturb to also make this true, let $\nu \rightarrow S$ be a tubular neighborhood $\nu \subset K_{0} \times I$ around $S \subset K_{0} \times I$. Using this we may canonically parameterize any small perturbation of $S$ as an embedded manifold by $S$. With this choice, any small Hamiltonian perturbation (in the interior of $I$ ) of the isotopy now gives rise to a perturbation of the function $p: S \rightarrow I$. As usual, we see that we can perturb in this way to make $p$ Morse, by arguing that there exists a family of such perturbation which makes the family of differentials $d p$ have zero as a regular value. This is possible, since for any point $(x, t) \in S$ where $d p=0$ we have that $K_{t}$ and $L$ have a tangential direction at $x$; and by introducing a Hamiltonian flow that rotates $K_{t}$ around the point $x$ (and the $K_{s}$ for $s$ close to $t$ ) we can make this transverse - in fact, the differential of $d p$ under this rotation is non-zero. Since this differential is non-zero in a small neighborhood, we may cover the compact set $d p=0$ with finitely many charts, and thus create a finite-dimensional family of perturbations which has $d p$ transverse to zero. It follows by the transversality theorem that for an open and dense set in the family the functions $p$ is Morse.

Consider an arbitrary member $K_{t}$ in the isotopy produced by Lemma 3.15. Choosing $\varepsilon$ sufficiently small, we may assume that $K_{t \pm \varepsilon} \cap L$ is arbitrarily close to $K_{t} \cap L$. In particular, since the intersection points are isolated, each intersection point of $K_{t \pm \varepsilon}$ with 
$L$ is close to a unique intersection point of $K_{t}$ with $L$. We obtain a decomposition

$$
K_{t \pm \varepsilon} \cap L=\bigcup_{x \in K_{t} \cap L} B_{x}^{ \pm}
$$

where $B_{x}^{ \pm}$consists of those intersection points which are close to $x$ (if $x$ were a generic intersection point we would conclude that $B_{x}^{ \pm}$is a singleton, but this is not the case in general).

Lemma 3.16. For each $t \in[0,1]$ and $\delta>0$ which is sufficiently small, there is a constant $\varepsilon$ such that

- $K_{t+\varepsilon}, K_{t-\varepsilon}$, and $L$ are pairwise transverse;

- $K_{t+\varepsilon}$ and $K_{t-\varepsilon}$ are $\delta$-close to $K_{t}$ and the action of each intersection point $z$ between $K_{t+\varepsilon}$ and $K_{t-\varepsilon}$ satisfies $|\mathcal{A}(z)| \ll \delta$;

- for any intersection point $x_{ \pm} \in B_{x}^{ \pm}$, the difference in actions satisfies

$$
\left|\mathcal{A}\left(x_{ \pm}\right)-\mathcal{A}(x)\right| \ll \delta
$$

Proof. Given any $t \in[0,1]$, we may, by condition (1) and (3) above, choose $\varepsilon$ sufficiently small so that the Lagrangians $\left(K_{t+\varepsilon}, L, K_{t-\varepsilon}\right)$ are pairwise transverse. Since the action and the distance to an intersection point of $L$ with $K_{t}$ are continuous in $\varepsilon$, the desired conditions can be achieved for $\varepsilon$ sufficiently small.

The above result, together with the monotonicity lemma, gives the following constraint on moduli spaces of holomorphic triangles.

Corollary 3.17. Given distinct intersection points $x$ and $y$ of $L$ and $K_{t}$, with $\mathcal{A}(x) \leqslant \mathcal{A}(y)$, and $\varepsilon$ sufficiently small, the moduli space $\mathcal{M}\left(x_{+}, z, y_{-}\right)$is empty for each $z \in K_{t-\varepsilon} \cap K_{t+\varepsilon}, x_{+} \in B_{x}^{+}$and $y_{-} \in B_{y}^{-}$. Similarly, for $\mathcal{A}(y) \leqslant \mathcal{A}(x)$ and $\mathcal{M}\left(y_{-}, z, x_{+}\right)$. Moreover, there is a contractible neighbourhood $U_{x}$ of each intersection point $x$ such that, if $x_{ \pm} \in B_{x}^{ \pm}$, then any element of $\mathcal{M}\left(x_{+}, z, x_{-}\right)$is homotopic to a map into $U_{x}$ relative the two marked points which map to $x_{+}$and $x_{-}$.

Proof. Choose disjoint neighbourhoods $U_{x}$ of the intersection points of $K_{t}$ with $L$, so that the conclusion of Lemma A.1 holds for $U_{x}$ and some $\delta>0$. Then, choose $\varepsilon$ small enough so that $B_{x}^{ \pm} \subset U_{x}$, and so that the conclusions of Lemma 3.16 hold for this $\delta$.

Stokes's theorem implies that the energy of any element of $\mathcal{M}\left(x_{+}, z, y_{-}\right)$is given by

$$
\mathcal{A}\left(x_{+}\right)-\mathcal{A}\left(y_{-}\right)-\mathcal{A}(z)=\left(\mathcal{A}\left(x_{+}\right)-\mathcal{A}(x)\right)+(\mathcal{A}(x)-\mathcal{A}(y))+\left(\mathcal{A}(y)-\mathcal{A}\left(y_{-}\right)\right)-\mathcal{A}(z) \ll \delta,
$$

where the second inequality follows from the conclusion of Lemma 3.16, and the assumption that $\mathcal{A}(x) \leqslant \mathcal{A}(y)$. On the other hand, Lemma A.1 implies that the energy must be 
greater than $\delta$ when $x \neq y$, so the moduli space is empty. Similar for the moduli space $\mathcal{M}\left(y_{-}, z, x_{+}\right)$when $\mathcal{A}(y) \leqslant \mathcal{A}(x)$.

The second statement follows from Corollary A.2.

Remark 3.18. The use of basic action estimates would be sufficient to prove the above result with the stronger assumptions that the inequality between the action of $x$ and $y$ is strict. The monotonicity lemma allows us to strengthen this to the case $\mathcal{A}(x)=\mathcal{A}(y)$ which will be crucial in the proof of the next result.

We now arrive at the main result of this section.

Theorem 2. If $K_{0}$ and $K_{1}$ are Hamiltonian isotopic, and $L$ is transverse to both, the equivalences

$$
\begin{aligned}
& C F_{*}\left(K_{0}, L ; R\right) \longrightarrow C F_{*}\left(K_{1}, L ; R\right) \\
& C F_{*}\left(L, K_{0} ; R\right) \longrightarrow C F_{*}\left(L, K_{1} ; R\right)
\end{aligned}
$$

have trivial Whitehead torsion.

Proof. As the arguments for the two maps are completely symmetric, we only address one of them.

Perturbing and subdividing any Hamiltonian isotopy into pieces as considered above, we can further subdivide into pieces as in the corollary above. We therefore start by proving that the equivalence in $C F^{*}\left(K_{t-\varepsilon}, K_{t+\varepsilon} ; \mathbb{Z}\right)$ induces a map

$$
C F_{*}\left(K_{t-\varepsilon}, L ; R\right) \longrightarrow C F_{*}\left(K_{t+\varepsilon}, L ; R\right)
$$

whose Whitehead torsion is trivial.

Consider the action filtration given by the critical values of the action functional. If $c_{0}<\ldots<c_{d}$ are the values of the action functional on $K_{t} \cap L$, we put $a(p)=-\frac{1}{2}\left(c_{p}+c_{p-1}\right)$ (with $a(0)>-c_{0}$ and $a(d+1)<-c_{d}$ ) and consider the corresponding action filtration. We write

$$
G^{p} C F_{*}\left(K_{t \pm \varepsilon}, L ; R\right) \equiv F^{a(p)} C F_{*}\left(K_{t \pm \varepsilon}, L ; R\right) / F^{a(p+1)} C F_{*}\left(K_{t \pm \varepsilon}, L ; R\right)
$$

for the associated graded group. By the description of moduli spaces in Corollary 3.17, the chain homotopy equivalence (induced by any Floer theoretical equivalence) is well defined on the quotients

$$
G^{p} C F_{*}\left(K_{t-\varepsilon}, L ; R\right) \longrightarrow G^{p} C F_{*}\left(K_{t+\varepsilon}, L ; R\right),
$$

and splits as a direct sum of maps of complexes of $R$-modules - one for each of the original intersection points in $K_{t} \cap L$ (this is where Remark 3.18 is relevant). 
Corollary 3.17 applied to the inverse shows that we have quasi-equivalences on the quotients. Moreover, there is a fixed contractible set which contains the image (up to homotopy relative the two marked points lying on $L$ ) of each holomorphic triangle which contributes to the map of associated graded spaces. This means that the map on these quotients induced by multiplying by the equivalence can be identified with the map

$$
G^{p} C F_{*}\left(K_{t-\varepsilon}, L ; \mathbb{Z}\right) \longrightarrow G^{p} C F_{*}\left(K_{t+\varepsilon}, L ; \mathbb{Z}\right)
$$

tensored with the identity on $R$. Since the Whitehead group of the trivial group is trivial and Whitehead torsion is functorial with respect to group homomorphisms - the complex with coefficients in $R$ also have trivial Whitehead torsion. So, Corollary 2.4 shows that the map in equation (3.28) has trivial torsion, from which we deduce the case of general isotopies by concatenation.

The above result has the following straightforward generalization.

Corollary 3.19. The Whitehead torsions of equation (3.21) and (3.22) are invariant under Hamiltonian isotopy of $K, L$ and $Q$.

Proof. Let $L_{0}$ and $L_{1}$ be Hamiltonian isotopic. As a special case of equation (3.19), we have a chain homotopy commutative diagram

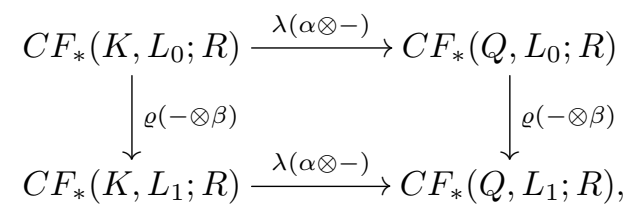

where the vertical maps are the maps considered in Theorem 2 which up to homotopy do not depend on the choice of $\beta \in C F^{0}\left(L_{0}, L_{1} ; \mathbb{Z}\right)$. Using Theorem 2, Lemma 2.5 and equation (2.1), we see that the two horizontal maps have the same Whitehead torsion.

Changing $K$ or $Q$ involves the following slightly different diagrams:
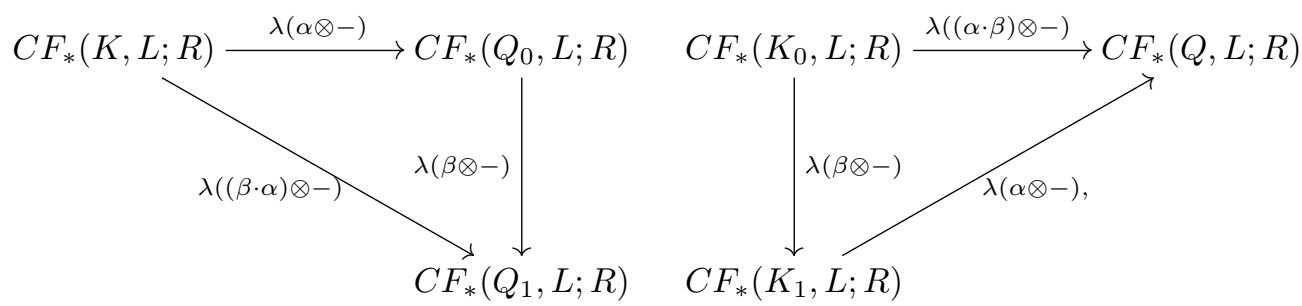

but the conclusion is the same since in each case the additional map (multiplication with $\beta$ ) has trivial torsion by Theorem 2 , and the diagrams homotopy commute by using equation (3.18) and its analogue for the left module action. 


\section{The Floer-theoretic torsion vanishes}

In this section we specialize to the setting of cotangent bundles: recall that, if $N$ is a closed manifold, every closed exact Lagrangian in $T^{*} N$ may be equipped with a relative Spin structure so that it is Floer-theoretically equivalent to the zero section ([14], [9], [2], [11]). Our main result will be the following.

Proposition 4.1. If $L \subset T^{*} N$ is an exact Lagrangian, then the torsion of the Floertheoretic equivalence with the zero section vanishes.

We shall prove this result by appealing to the Hamiltonian invariance of torsion and a large-scale deformation of the Lagrangian $L$. The deformation of $L$ is essentially the same as that used in [12].

Denote by $\psi^{r}$ the time- $(\log r)$ Liouville flow (which corresponds to dilating the fibres by $r$ ), and by $\phi_{g}$ the time- 1 Hamiltonian flow of $g \circ q$, where $g: N \rightarrow \mathbb{R}$ is a Morse function with distinct critical values. For any $K \subset T^{*} N$ we define

$$
K^{r}=\psi^{r} K \quad \text { and } \quad K_{1}^{r}=\phi_{g} K^{r}
$$

and abbreviate $N_{1}^{r}$ as $N_{1}$, since the zero section is invariant under the Liouville flow. Note that even though the Liouville flow is not Hamiltonian, we have the following result, which follows via an elementary computation with the Liouville form.

Lemma 4.2. If $K$ is an exact Lagrangian of $T^{*} N$ with primitive $f: K \rightarrow \mathbb{R}$, then the isotopy from $K$ to $K^{r}$ and $K_{1}^{r}$ is Hamiltonian, and equips these Lagrangians with primitives $r f$ and $r f+g$ under the identification with $K$ induced by the isotopy.

Corollary 3.19 implies that the Floer theoretic torsion of the equivalence of $L$ with the zero section agrees with the torsion of the map

$$
C F_{*}\left(L^{r}, L_{1}^{r} ; R\right) \longrightarrow C F_{*}\left(N, N_{1} ; R\right),
$$

induced by left and right multiplication with Floer theoretic equivalences $\alpha^{r} \in C F^{*}\left(L^{r}, N\right)$ and $\beta_{1}^{r} \in C F^{*}\left(N_{1}, L_{1}^{r}\right)$.

By construction, intersection points of $N$ and $N_{1}$ occur at critical points of $g$, and $\mathcal{A}(x)=g(x)$. In particular, if $c_{0}<\ldots<c_{d}$ are the critical values of $g$, then we consider the action filtration

$$
F^{a(p)} C F_{*}\left(N, N_{1} ; R\right)
$$

using the actions $a(p)=-\frac{1}{2}\left(c_{p}+c_{p-1}\right)$; the fact that there is a unique intersection $x_{p}$ of action $c_{p}$ implies that the associated graded group is

$$
G^{p} C F_{*}\left(N, N_{1} ; R\right) \cong R_{x_{p}}
$$


We shall now assume that $r \ll 1$, in which case the following statements hold:

- the cocycles $\alpha^{r}$ and $\beta_{1}^{r}$ and their quasi-inverses are supported in action level very close to zero (using the primitives from Lemma 4.2);

- the intersection points of $L^{r}$ and $L_{1}^{r}$ decompose as a disjoint union

$$
L^{r} \cap L_{1}^{r}=\coprod_{p=0}^{d} B_{p}^{r}
$$

with $B_{p}^{r}$ consisting of those intersection points lying in a small neighbourhood of the critical point $x_{p}$ of $g$.

(i) The action of any element $y \in B_{p}^{r}$ satisfies

$$
\left|\mathcal{A}(y)-c_{p}\right| \ll 1
$$

(again by the explicit primitives from Lemma 4.2).

We now consider the filtration on $C F_{*}\left(L^{r}, L_{1}^{r} ; R\right)$ given by the same action levels as the above filtration on $C F_{*}\left(N, N_{1} ; R\right)$. The $p$ th associated graded group is generated by intersection points of action between $\frac{1}{2}\left(c_{p}+c_{p-1}\right)$ and $\frac{1}{2}\left(c_{p+1}+c_{p}\right)$. By equation (4.6), all such intersection points in fact have action approximately equal to $c_{p}$ and lie in the set $B_{p}^{r}$ of intersection points which are close to $x_{p}$. This associated graded group is therefore given by

$$
G^{p} C F_{*}\left(L^{r}, L_{1}^{r} ; R\right) \cong \bigoplus_{y \in B_{p}^{r}} R_{y},
$$

and is equipped with the induced differential.

Since left and right multiplication induce maps which preserve action filtrations, and the equivalences $\alpha^{r}$ and $\beta_{1}^{r}$ have small action, we conclude that equation (4.2) descends to the associated graded with respect to the filtration, as does the chain homotopy inverse induced by multiplication with the quasi-inverses of $\alpha^{r}$ and $\beta_{1}^{r}$. We conclude the following.

LEMMA 4.3. The equivalences $\alpha^{r}$ and $\beta_{1}^{r}$ induce a chain homotopy equivalence

$$
G^{p} C F_{*}\left(L^{r}, L_{1}^{r} ; R\right) \longrightarrow G^{p} C F_{*}\left(N, N_{1} ; R\right)
$$

On the other hand, equation (4.8) agrees with the map obtained from

$$
G^{p} C F_{*}\left(L^{r}, L_{1}^{r} ; \mathbb{Z}\right) \longrightarrow G^{p} C F_{*}\left(N, N_{1} ; \mathbb{Z}\right)
$$

by extension of scalars from $\mathbb{Z}$ to $R$. Indeed, using Corollary A.2 with $K=N$ and $Q=N_{1}$, we see (as in the proof of Theorem 2) that the homotopy class of the path defined by 


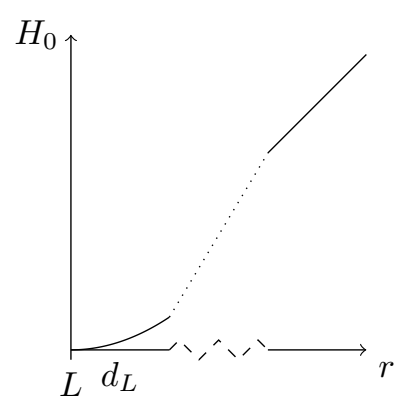

Figure 7. The function $H$ approximates.

any rigid disc counted in these maps is homotopic relative to end points to a path inside a small contractible neighbourhood of $x_{p}$.

In particular, the Whitehead torsion of equation (4.8) vanishes. Since this is the map on associated graded groups of equation (4.2), we conclude from Corollary 2.4 that the Whitehead torsion vanishes. Using invariance of torsion under Hamiltonian isotopies, we arrive at the main result of this section.

Proposition 4.4. The Whitehead torsion of any Floer-theoretic equivalence of closed exact Lagrangians in $T^{*} N$ vanishes.

\section{From cellular to Floer cochains}

In this section, we prove that the Whitehead torsion of the projection map $L \rightarrow N$, which we call the classical torsion, agrees with the Floer theoretic torsion. We will prove this by passing through a sequence of intermediary maps which are defined using Morse and Floer theory in the disc cotangent bundle $D^{*} N \subset T^{*} N$ (which we assume contains $L$ in its interior).

On the Morse theoretic side, we consider a Morse function $H$ which $C^{1}$-approximates a function

$$
H_{0}(z)= \begin{cases}d_{L}(z)^{2}, & \text { if } d_{L}(z)<\varepsilon \\ r, & \text { if } r>1-\varepsilon\end{cases}
$$

Here $d_{L}: D^{*} N \rightarrow \mathbb{R}$ is the distance function to $L$ in some Riemannian metric we fix such that $L$ is a totally geodesic submanifold, and $r: D^{*} N \rightarrow[0,1]$ is the radial coordinate equal to 1 on the boundary $\partial D^{*} N$. The function values elsewhere will not matter and we assume that $\varepsilon$ is so small that this is well defined and smooth. This function is illustrated in Figure 7. To perturb this close to $L$, we consider a small positive Morse 
function $h: L \rightarrow \mathbb{R}$ and assume that in a small neighbourhood of $L$ we have

$$
H(z)=h(\pi(z))+d_{L}(z)^{2}
$$

where $\pi(z)$ is the nearest point to $z$ in $L$. Note that $h=\left.H\right|_{L}$ and, by the assumption on the Riemannian structure, the critical points of $H$ which lie on $L$ have descending manifolds contained in $L$, which agree with the descending manifolds of the corresponding critical points of $h$. Close to the boundary $\partial D^{*} N$, we assume that $H$ has outwards pointing gradient (as $H_{0}$ does) and that it is a Morse function when restricted to the boundary. We conclude that the Morse complex $C M_{*}(L, h ; R)$ embeds as a subcomplex into $C M_{*}\left(D^{*} N, H ; R\right)$.

Lemma 5.1. The classical torsion agrees with the torsion of the inclusion

$$
C M_{*}(L ; h, R) \rightarrow C M_{*}\left(D^{*} N ; H, R\right) .
$$

Proof. We first use the factorization

$$
L \longrightarrow D^{*} N \longrightarrow N
$$

to see that the classical torsion agrees with the torsion of the map $L \rightarrow D^{*} N$ we note that the second map has trivial torsion, since $D^{*} N$ has a sequence of simple collapses to $N$.

We now appeal to the fact that for a generic Riemannian metric on $D^{*} N$ the union of the descending manifolds of $H$ yield a CW subcomplex $X_{D^{*} N} \rightarrow D^{*} N$. Picking the metric so that $L$ is totally geodesic, we obtain a further factorization

$$
L \rightarrow X_{D^{*} N} \rightarrow D^{*} N
$$

Using the explicit way $H$ looks close to the boundary, we may compare the latter two by building an even bigger CW complex with cells given by critical points of $H$ in the interior and two for each critical point of $H$ restricted to $\partial D^{*} N$ (which we assumed to be Morse). That is, first we build $X_{D^{*} N}$, then we disjointly build $\partial D^{*} N$ and then finally for each cell in the boundary we can use the descending manifold inside the boundary together with the directions into $D^{*} N$ to attach a new cell of one dimension higher than the cell in the boundary. This is homeomorphic to $D^{*} N$ and shows that the inclusion $X_{D^{*} N} \rightarrow D^{*} N$ has trivial torsion. Indeed, the difference of the two cell structures is a sequence of trivial collapses from $D^{*} N$ to $X_{D^{*} N}$ by starting with the top-dimensional cells (associated with critical points of the boundary Morse function) and going down in dimension. Combining the above arguments, we conclude that the torsion of $L \rightarrow N$ agrees with the torsion of $L \rightarrow X_{D^{*} N}$. 


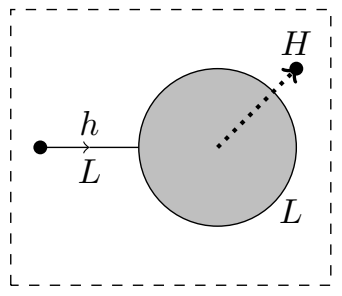

Figure 8. Standard Morse map in Lemma 5.1 interpreted as a count of discs and gradient flow lines: the dotted line is a flow line of $H$ starting at the centre of the disc and converging to a critical point.

To complete the argument, we observe that the map $L \rightarrow X_{D^{*} N}$ is cellular with respect to the Morse-theoretic $\mathrm{CW}$ structure on $L$, and we have a natural commutative diagram

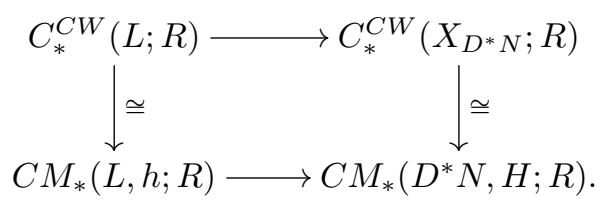

We have arranged our fixed choice of pair of Morse functions so that equation (5.2) is an inclusion. This inclusion can also be interpreted as a count of configurations shown in Figure 8, which consist of (1) a flow line $\gamma_{-}$of $h$ parameterized by $(-\infty, 0],(2)$ a flow line $\gamma_{+}$of $H$ parameterized by $[0, \infty)$, and (3) a holomorphic map $u: D^{2} \rightarrow M$ mapping the boundary to $L$, such that $u(-1)=\gamma_{-}(0)$ and $u(0)=\gamma_{+}(0)$. The key point is that all such rigid configurations are constant: the exactness of $L$ implies that all holomorphic discs are constant, and equation (5.1) implies that all negative gradient flow lines of $H$ starting on $L$ are contained in $L$.

Proposition 5.2. The classical and Floer-theoretic torsions agree.

Proof. Consider the composition

$$
C M_{*}(L ; h, R) \stackrel{\varrho}{\longrightarrow} C F_{*}(L, N ; R) \stackrel{\lambda}{\longrightarrow} C M_{*}(N, N ; R) \stackrel{m}{\longrightarrow} C M_{*}\left(D^{*} N ; H, R\right) .
$$

The last map $m$ is the map considered in Lemma 5.1 (in the case $L=N$ ) and therefore has trivial Whitehead torsion. Indeed, the standard inclusion $N \subset D^{*} N$ has trivial Whitehead torsion. The proposition will thus follow from the claim that the composition of these is homotopic to the map from Lemma 5.1, which is represented in Figure 8.

Let $\alpha \in C F^{*}(L, N ; \mathbb{Z})$ and $\beta \in C F^{*}(N, L ; \mathbb{Z})$ be Floer cochains inducing the Floer theoretic equivalences, and let $f: N \rightarrow \mathbb{R}$ be any Morse function on $N$ whose stable manifolds are transverse to the unstable manifolds of $H$. The maps in the sequence are given by counting rigid objects in moduli spaces of maps as in Figure 9. 


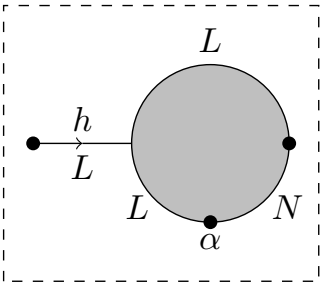

$\varrho$

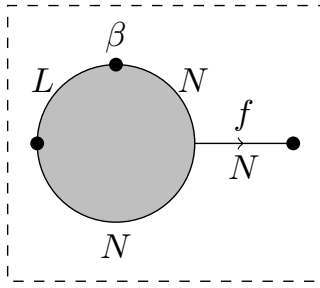

$\lambda$

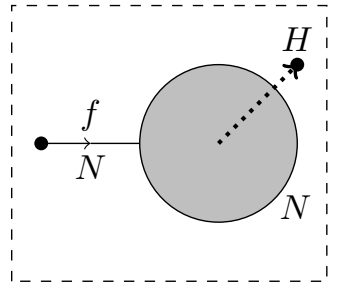

$m$

Figure 9. The three maps in equation (5.6).

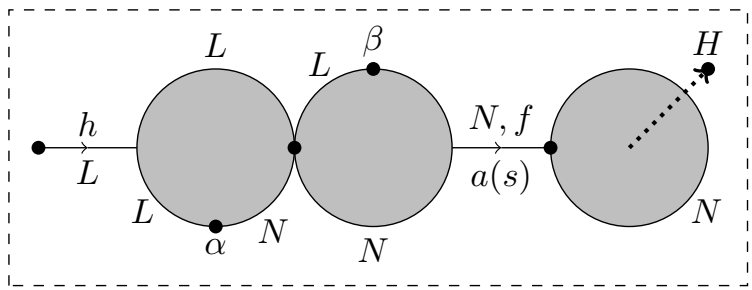

Figure 10. The moduli space for $-1<s<0$.

Here marked points on the boundary of a disc should be mapped to an intersection point.

To create a homotopy between the two maps we construct a 1-parameter family of moduli spaces, parameterized by $s \in[-1,1]$, interpolating between Figures 8 and 9 .

- Near $s=-1$, we glue the flow lines in the right and centre of Figure 9 to obtain a (finite of length $a(s)$ ) flow line of $f$ on $N$. The resulting configuration of discs and flow lines is illustrated in Figure 10.

- As $s$ approaches zero from the left, we let the length of the finite gradient flow line along $N$ go to zero. In particular, at $s=0$, we obtain the moduli space shown in Figure 11 which has three disc components meeting at two boundary nodes. These three disc components represent a point in (the Gromov boundary of) the moduli space of discs with three boundary marked points and one interior marked point. This moduli space is connected and has dimension 2 .

- For $0<s<1$ we pick a path in the moduli space of such discs, as shown in Figure 12, between the configuration appearing at $s=0$, and the configuration shown in Figure 13 consisting of a stable disc with two components meeting at a node, so that one disc is biholomorphic to the unit disc $D^{2} \subset \mathbb{C}$ with boundary marked points at $(-1,1)$ and an interior marked point at the origin, and the other is a disc with three marked points, one of which is the node, and the others are labeled by the equivalences $\alpha$ and $\beta$.

In this way, we have produced a homotopy between the composition appearing in 


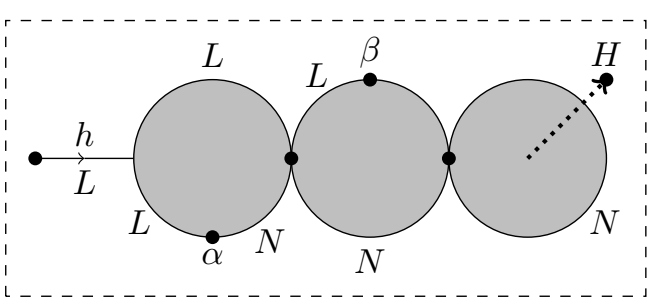

Figure 11. The moduli space for $s=0$.

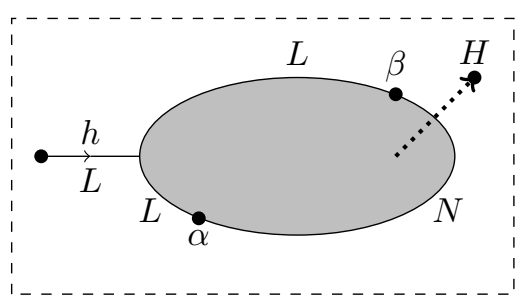

Figure 12. The moduli space at $0<s<1$.

equation (5.6) and the map defined by the moduli spaces for $s=1$. Since $\alpha$ and $\beta$ are Floer theoretic equivalences, the (signed) count of holomorphic bigons with corners labeled $\alpha$ and $\beta$ passing thought a generic point of $L$ is 1 . This implies that the (signed) count of configurations shown in Figure 13 is the same as the count of the moduli space appearing in Figure 8, which, by Lemma 5.1, agrees with the classical torsion. Note in particular that each contribution to the local coefficient system is the same, since this is given by the path (unique up to homotopy) from the far left of the diagram to the marked point at the end of the arrow decorated with $H$.

Remark 5.3. If we did not specify the modulus of the disc with interior marked point in Figure 13, the corresponding moduli space would have excess dimension, corresponding to the fact that the boundary of a disc with interior marked point sweeps a 1-dimensional cycle in $L$, and hence could not rigidly intersect the $n$-dimensional cycle in $L$, which accounts for the product of $\alpha$ and $\beta$. We obtain a rigid intersection by specifying that the disc is biholomorphic to one with marked points at \pm 1 and the origin. Any other choice of modulus would give rise to a homotopic map.

\section{Appendix A. The monotonicity lemma}

Let $M$ be any symplectic manifold (possibly non-compact) with a given almost complex structure $J$, and let $K$ and $Q$ be two proper Lagrangians in $M$ with an isolated intersection point $x \in K \cap Q$. 


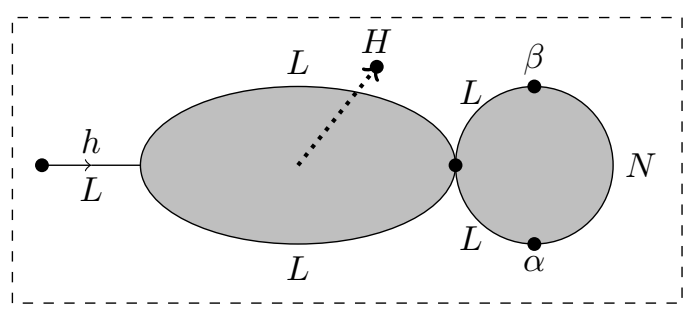

Figure 13. The moduli space for $s=1$.

Let $U_{x}$ be a neighbourhood of $x$, and consider $J$-holomorphic discs $u$ : $D^{2} \rightarrow M$ which satisfy the following conditions with respect to a constant $\delta>0$ :

$$
u(1) \in U_{x} \quad \text { and } \quad u(-1) \notin U_{x}
$$

$u$ maps the upper semi-circle in $\partial D^{2}$ to a $\delta$ neighbourhood of $K$,

and the lower semi-circle to a $\delta$ neighbourhood of $Q$.

The following technical result about minimal areas of holomorphic curves is a variant of the monotonicity lemma, and is crucially used in $\S 3$ and $\S 4$.

Lemma A.1. For each sufficiently small neighbourhood $U_{x}$ there is a constant $\delta$ such that all holomorphic discs satisfying conditions (A.1) and (A.2) have area greater than $\delta$.

Proof. Pick a Darboux chart $D_{\varepsilon}^{2 n} \rightarrow M$ mapping the origin to $x$ such that $Q$ is identified with $D_{\varepsilon}^{n}$, and such that the image of the tangent space of $K$ at $x$ has trivial intersection with $i \mathbb{R}^{n}$ (i.e. is non-vertical). Locally $K$ is given by the differential of a function, and, by making $\varepsilon$ smaller, we may assume that $f: D_{\varepsilon}^{n} \rightarrow \mathbb{R}$ is such a function, and that the differential defines $K$ in a neighbourhood of type $D_{\varepsilon}^{n} \times i D_{\varepsilon^{\prime}}^{n}$.

Since $f$ has an isolated critical point at zero, we have a codimension- 2 manifold

$$
W=\left\{(q, p) \in D_{\varepsilon}^{n} \times i D_{\varepsilon^{\prime}}^{n}:\|q\|=\frac{1}{2} \varepsilon \text { and }\|p\|=\frac{1}{2}\left\|d_{q} f\right\|\right\},
$$

which is an $S^{n-1}$ bundle over the sphere of radius $\frac{1}{2} \varepsilon$ in $\mathbb{R}^{n}$.

We shall presently appeal to the monotonicity lemma [20, Proposition 4.3.1(ii)]: there is a constant $C_{4}$ such that any holomorphic curve which intersects $W$ and has boundary in the complement of the $r$ neighbourhood of $W$ has area greater than $C_{4} r^{2}$, whenever $r$ is sufficiently small.

Let us therefore choose some neighbourhood $U$ of $x$ inside the chart whose closure is disjoint from $W$, and pick $\delta$ so that the following properties hold in the Darboux chart:

(1) the $\delta$ neighbourhoods of $Q$ and $K$ only have common points in $U$;

(2) the $r$-neighbourhood of $W$, with $r=\sqrt{\delta / C_{4}}$, is disjoint from $U$ and from the $\delta$-neighbourhoods of $Q$ and $K$, and this $r$ is small enough for the monotonicity lemma to apply. 
The first assumption implies that $u(-1)$ lies outside of the chart. The image of the boundary of $u$ under the collapse map $M \rightarrow D_{\varepsilon}^{2 n} / \partial D_{\varepsilon}^{2 n}$ is therefore a loop which winds once around $W$. The algebraic intersection number of $u$ with $W$ is hence non-trivial modulo 2. The image of $u$ therefore intersects $W$, while the image of its boundary lies outside the $\delta$-neighbourhood of $W$. The monotonicity lemma implies that such a curve has area greater than $\delta$, completing the proof.

We shall also consider the following variant.

Corollary A.2. For each sufficiently small neighbourhood of $x$ there is a constant $\delta$ such that, if condition (A.2) holds, the image of \pm 1 under $u$ lies in $U_{x}$, and the area of $u$ is less than $\delta$, then the path $\{u(t)\}_{t \in[-1,1]}$ is homotopic relative to its end points to a map with image in $U_{x}$.

Proof. Assume by contradiction that there is such a disc for which the path from $u(-1)$ to $u(1)$ is not homotopic to a path with image in $U_{x}$, we then see that the lift of $u$ to the universal cover of $M$ (with the canonical symplectic structure) has endpoints mapping to distinct inverse images of $U_{x}$. Replacing $K$ and $Q$ by their inverse images, we find a contradiction to the previous lemma and the fact that lifting any pseudo-holomorphic disc preserves the symplectic area.

\section{References}

[1] Abouzaid, M., A cotangent fibre generates the Fukaya category. Adv. Math., 228 (2011), 894-939.

[2] - Nearby Lagrangians with vanishing Maslov class are homotopy equivalent. Invent. Math., 189 (2012), 251-313.

[3] Акано, M. \& Joyce, D., Immersed Lagrangian Floer theory. J. Differential Geom., 86 (2010), 381-500.

[4] AтiYah, M. F., Thom complexes. Proc. London Math. Soc., 11 (1961), 291-310.

[5] Barraud, J.-F. \& Cornea, O., Lagrangian intersections and the Serre spectral sequence. Ann. of Math., 166 (2007), 657-722.

[6] Biran, P. \& Cornea, O., Rigidity and uniruling for Lagrangian submanifolds. Geom. Topol., 13 (2009), 2881-2989.

[7] Damian, M., Floer homology on the universal cover, Audin's conjecture and other constraints on Lagrangian submanifolds. Comment. Math. Helv., 87 (2012), 433-462.

[8] Floer, A., Morse theory for Lagrangian intersections. J. Differential Geom., 28 (1988), 513-547.

[9] Fukaya, K., Seidel, P. \& Smith, I., Exact Lagrangian submanifolds in simply-connected cotangent bundles. Invent. Math., 172 (2008), 1-27.

[10] Hutchings, M. \& Lee, Y. J., Circle-valued Morse theory and Reidemeister torsion. Geom. Topol., 3 (1999), 369-396.

[11] Kragh, T., Parametrized ring-spectra and the nearby Lagrangian conjecture. Geom. Topol., 17 (2013), 639-731. 
[12] - Homotopy equivalence of nearby Lagrangians and the Serre spectral sequence. Math. Ann., 368 (2017), 945-970.

[13] Milnor, J., Whitehead torsion. Bull. Amer. Math. Soc., 72 (1966), 358-426.

[14] Nadler, D., Microlocal branes are constructible sheaves. Selecta Math., 15 (2009), 563619.

[15] Nash, J., A path space and the Stiefel-Whitney classes. Proc. Nat. Acad. Sci. U.S.A., 41 (1955), 320-321.

[16] Piunikhin, S., Salamon, D. \& Schwarz, M., Symplectic Floer-Donaldson theory and quantum cohomology, in Contact and Symplectic Geometry (Cambridge, 1994), Publ. Newton Inst., 8, pp. 171-200. Cambridge Univ. Press, Cambridge, 1996.

[17] Seidel, P., Fukaya Categories and Picard-Lefschetz Theory. Zurich Lectures in Advanced Mathematics. Eur. Math. Soc. (EMS), Zürich, 2008.

[18] - Homological mirror symmetry for the genus two curve. J. Algebraic Geom., 20 (2011), $727-769$.

[19] Sheridan, N., Homological mirror symmetry for Calabi-Yau hypersurfaces in projective space. Invent. Math., 199 (2015), 1-186.

[20] Sikorav, J.-C., Some properties of holomorphic curves in almost complex manifolds, in Holomorphic Curves in Symplectic Geometry, Progr. Math., 117, pp. 165-189. Birkhäuser, Basel, 1994.

[21] SuÁrez, L. S., Exact Lagrangian cobordism and pseudo-isotopy. Internat. J. Math., 28 (2017), 1750059, 35 pp.

[22] Sullivan, M. G., K-theoretic invariants for Floer homology. Geom. Funct. Anal., 12 (2002), 810-872.

[23] Тном, R., Espaces fibrés en sphères et carrés de Steenrod. Ann. Sci. École Norm. Sup., 69 (1952), 109-182.

[24] Whitehead, J. H. C., Simple homotopy types. Amer. J. Math., 72 (1950), 1-57.

Mohammed Abouzaid

Department of Mathematics

Columbia University

Room 509, MC 4406

2990 Broadway

New York, NY 10027

U.S.A.

abouzaid@math.columbia.edu

Received March 17, 2016

Received in revised form October 23, 2017
Thomas Kragh

Department of Mathematics

Uppsala University

PO Box 480

SE-751 06 Uppsala

Sweden

thomas.kragh@math.uu.se 